\title{
3D Pottery Shape Matching Using Depth Map Images
}

\author{
Anestis Koutsoudis \\ akoutsou@ceti.gr \\ Christodoulos Chamzas \\ chamzas@ceti.gr \\ Cultural and Educational Technology Institute/Research Centre 'ATHENA', Tsimiski 58, Xanthi, 67100, \\ Greece
}

\begin{abstract}
The utilisation of 3D computer graphics technologies in the domain of pottery analysis can enhance archaeological research in terms of data management, indexing and shape matching. In this paper, we attempt to reduce the dimensions of the $3 \mathrm{D}$ vessel shape matching problem in order to create Web-enabled compact shape descriptors applicable for content based retrieval systems. This is achieved by exploiting significant morphological features of vessels such as the rotational symmetry and the opposed positioning of appendages. We propose the idea of capturing the surface relief of a normalised, in terms of scale, position and orientation 3D vessel on a pair of depthmap images. We question the ability of performing shape matching of complete or nearly complete 3D vessels by encoding depthmap images using 2D shape descriptors. We have evaluated the performance of two shape descriptors applied on depthmap images in relation to a generic 3D shape descriptor. For the performance evaluation, we have implemented multiple queries-by-example on five ancient Greek vessel shape categories found in our calibrated 3D vessel database.
\end{abstract}

\section{Keywords: 3D Pottery/Shape matching/Content Based Retrieval/Zernike moments/Vessels}

\section{Introduction}

The use of 3D content based retrieval (3DCBR) mechanisms can provide efficient management of $3 \mathrm{D}$ models in terms of indexing, searching and retrieving [1][2]. Although keyword-based search engines have made progress over the last decade [3][4], 
in situations where morphological features need to be described, keywords are not efficient for formulating such complex queries [5]. 3DCBR technology allows the substitution of keywords by the actual 3D data. Such an approach introduces an intuitive approach for depicting the user's criteria and constraints in mind. Relying only on morphological properties, 3DCBR overcomes the multi-language barrier introduced by metadata and allows the discovery of supra-regional typology coherencies [6]. In general, archaeologists who are commencing research on pottery classification and analysis can be benefited from 3DCBR as the detection of shape similarities is one of their main objectives.

In this work, we exploit two of the main morphological features of 3D vessel replicas in order to create compact shape descriptors for web-based content based retrieval search engines. These features are the rotational symmetry and the opposed positioning of appendages. The work presented in this paper is an attempt towards the complexity reduction of the pottery shape matching problem by applying shape descriptors derived from the digital image domain. More specifically, we depict the outer surface relief of a $3 \mathrm{D}$ vessel's main body and appendages by using a pair of depthmap images. This is performed after applying our previously published scale, position and orientation normalisation algorithm [7]. Each depthmap contains an orthogonal projection of the vessel captured from a specific viewpoint. We consider that the information captured by the proposed viewpoints is sufficient for shape matching due to the rotational symmetry and the opposed positioning of appendages. We question the shape discrimination performance of three descriptors on a calibrated 3D pottery database. These are the following:

1. 2D Zernike moments of $13^{\text {th }}$ order.

2. A variant of the descriptor proposed by Chaouch et al. [8].

3. The generic LightField 3D shape descriptor proposed by Chen et al. [9]. 
The first two descriptors were used to encode the depthmap images while the third descriptor uses a different multiple viewpoint 3D shape encoding approach. We have evaluated the performance of these descriptors by executing multiple queries-by-example on our calibrated 3D pottery database which contains a total of $1,0123 \mathrm{D}$ vessels.

The subsequent sections of this paper are organized as follow: In Section 2, we present some related work while in Sections 3 and 4, we describe the depthmap extraction and the descriptor encoding procedures. In Section 5, we discuss the shape discrimination performance of the three descriptors and present objective quantitative results by using precision-recall graphs and performance scalars that reveal different performance aspects. Finally, we conclude in Section 6 by giving some thoughts on the current results and the future evaluation of other 2D shape descriptors.

\section{Related Work}

Several experimental software tools and methodologies, found in literature, attempt to enhance the archaeological research and study of pottery. Those can be organised in groups that use data derived from $2 \mathrm{D}$ profile images, complete or incomplete $3 \mathrm{D}$ vessel replicas and 3D shreds.

The reassembly problem of pots from 3D digitised shreds has piqued the interest of several researchers who attempted to reconstruct the main body of axially symmetric vessels [10]-[16]. Dezhi et al. [17][18] proposed the description of a vessel's profile using an XML based encoding scheme to support scientific information sharing over the Web. They proposed a set of profile properties such as end points, points of vertical tangency, corner points to describe the profile of a vessel that carries no appendages. Gilboa et al. [19] described a typology classification software tool for Iron Age bowls of Tel Dor. An 
interactive vessel drawing tool was proposed by Melero et al. [20]. Uppalapati et al. [21] described an interface that allows researchers to remotely access vessel collections and perform queries by matching sketches with a 2D curve matching algorithm. Similarly, Koutsoudis et al. [7] created a Web-based 3D pottery search engine where the user can perform queries-by-sketch. Hofer et al. [22] described a method that allows the recognition of 3D shapes using line element geometry. Chyr et al. [23] and Chen et al. [9] described two generic 3D shape matching methods that are based on the fact that objects that are visually similar from different viewpoints should carry similar morphological features. Sebastian et al. [24] proposed a method of generic 2D shape outline matching which could be also applied for the matching of $2 \mathrm{D}$ vessel profiles.

In general, shape description and shape matching are two interlinked problems that many research domains can benefit from. Iyer et al. [25] indicated that research trends are moved towards the generation of application-driven benchmark databases, the creation of domain specific descriptors that encapsulate domain knowledge and optimised shape representations.

To this end, we present a novel application of our previously published vessel specific scale, position and orientation normalisation algorithm [7]. Having a complete or nearly complete 3D vessel replica normalised by the algorithm described in [7], we question the possibility of using $2 \mathrm{D}$ shape descriptors for content based retrieval of $3 \mathrm{D}$ vessels. We attempt to exploit both axial symmetry and appendage positioning in order to reduce the dimensions of the $3 \mathrm{D}$ vessel mesh models shape description and matching problem and thus to provide compact shape descriptors applicable for Web-enabled search engines. As the number of $3 \mathrm{D}$ vessels replicas on the Web is continuously increased, a compact $3 \mathrm{D}$ vessel mesh model descriptor would allow the realisation of 3DCBR over the Web without the need of exploiting any additional data such as 2D profile images. 


\section{Depthmap based 3D vessel's surface relief capture}

The proposed methodology is based on the assumption that the main morphological features of a vessel's surface relief can be captured on a pair of depthmap images. The extraction of the depthmap images is performed after the completion of the automated scale, position and orientation normalisation algorithm [7]. In brief, the algorithm is applicable to complete or nearly complete triangular mesh 3D vessel replicas and involves the application of sequential 3D affine transforms in order to pose, orient and scale the vessel in 3D space. The affine transforms are performed by taking under consideration the vessel's axis of symmetry, appendages and mouth positioning.

Once the 3D mesh of a vessel is normalised, two orthographic projection virtual cameras are positioned on the positive sides of the Cartesian $X$ and $Z$ axes with the $Y$ axis coordinate equal to zero. Figure 1.a illustrates the virtual camera positioning. Each depthmap contains enough information to reconstruct the visible-from-the-viewpoint part of the vessel as a triangulated 3D mesh (Figure 1.b). The viewpoint normal vector of each camera points towards the vessel's axis of symmetry which is parallel to the Cartesian $Y$ axis. Additionally, the projection plane of each virtual camera covers an area equal to the side of a unit cube. In fact, the two projection planes are considered as two neighbouring unit cube planes. By using the depthmap rendering technique the surface relief of the $3 \mathrm{D}$ mesh is transformed into a $2 \mathrm{D}$ bitmap image with greyscale pixel values. The surface relief areas which are not captured by the virtual cameras are considered complementary to those been captured. This is due to the rotational symmetry of a vessel and the tubular structure of appendages. Hence a single depth map image captured from the Cartesian $Z$ axis might also be adequate for vessels with no appendages. Nevertheless, the use of the second depthmap image is proposed in order to capture the details found in vessels that carry appendages.

Figure 1 
We have implemented a stand alone application using Borland Delphi and the GLScene and VTK libraries in order to automatically extract the depthmap image pairs from a total of 1,012 vessels (94 3D digitised and 918 computer generated) found in our 3D pottery database.

\section{Depthmap image pair encoding}

Once the depthmap image pairs were extracted, they were encoded using two shape descriptors.

We have selected the $2 D$ Zernike moments (ZMDM) which is a region-based shape encoding descriptor that has been widely used due to its significant properties such as noise resilience, information redundancy and reconstruction capability [26][27]. Zernike moments encode a bitmap image by using a set of complex polynomials which define a complete orthogonal set over a unit disk and are invariant to rotations. If $f(i j)$ is the function of a bitmap image with $m x n$ dimensions then the $n$-th order Zernike moment with a repetition $l$ is given by $Z_{n l}=\frac{n+1}{\pi} \sum_{i=0}^{m-1} \sum_{j=0}^{n-1} f(i, j) \cdot R_{n l}\left(r_{i j}\right) \cdot e^{-x \cdot l \cdot \theta_{i j}}$ where the discrete polar coordinates are calculated by $r_{i j}=\sqrt{x_{j}^{2}+y_{i}^{2}}, \theta_{i j}=\arctan \left(\frac{y_{i}}{x_{i}}\right)$. The absolute values of the produced vector are rotation-invariant when the centre of the image is considered the origin of the unit disc. In our case this is true due to the fact that the $3 \mathrm{D}$ vessels are scaled normalised within a unit bounding sphere [7]. Therefore, the centre of each depthmap image is considered as an orthogonal projection of the centre of the sphere. In our experiments we have used the $13^{\text {th }}$ order Zernike moments which has already been found to be an optimum between the descriptor's vector dimensions and its shape discrimination ability [28]. Thus, the ZMDM descriptor vector is composed by 112 moment values where each depthmap contributes 56 moment values. The degree of similarity between two vessels is given by the L2-norm. 
On the other hand we have encoded the depthmap images using a variant of the descriptor proposed by Chaouch et al. [8]. Instead of following the initial generic method of encoding multiple depthmap images captured from viewpoints located on the facets of a bounding dodecahedron[8]; we applied the proposed character based encoding scheme on the depthmap image pairs of each 3D vessel. The Character Based Depth Map Encoding descriptor (CBDM) transforms each depthmap into a sequence of characters. Initially two predefined characters are used to discriminate between the vessel's surface region and the background region. Thus, a pixel that belongs to the outer-background region is represented by the $b$ character while the $i$ character represents a part of the innerbackground region (e.g. background areas surrounded by a handle). Furthermore, the vessel's surface region is encoded using a set of predefined characters that represent the surface's curvature direction (Figure 2). In our implementation we have used characters $u, d$ and $s$ which represent a positive line slope (going upwards), a negative line slope (going downwards) and a zero line slope (steady direction). The surface slope state is computed as the difference between two sequential pixels. Each image is encoded by following a row-by-row and a column-by-column approach. All row-based and columnbased character sequences are merged (Character string addition) together producing two cumulative character sequences $\left(C S_{\text {row }}\right.$ and $\left.C S_{\text {column }}\right)$. Thus each depthmap is represented by two character sequences. The degree of similarity between two vessels is given by the Needleman-Wunsch $(N W)$ global sequence alignment algorithm [29]. In our experiments, the total dissimilarity score $D$ between two vessels $i, j$ is given by summing up the $N W$ similarity scores of the cumulative character sequences: $D_{i, j}=\left[N W\left(C S_{\text {row }}^{i}, C S_{\text {row }}^{j}\right)+N W\left(C S_{\text {column }}^{i}, C S_{\text {column }}^{j}\right)\right]$.

Figure 2 


\section{Descriptors performance evaluation}

In order to verify our initial assumption of being able to capture the main morphological features of a 3D vessel on a pair of depthmap images we have selected to evaluate the performance of the ZMDM and CBDM descriptors in relation with a 3D shape descriptor that exploits the idea that two 3D objects are similar if they are visually similar. The Lightfield $3 D$ descriptor (LIFD) is a generic robust 3DCBR approach with high discrimination capabilities [23]. The LIFD descriptor encodes one hundred binary images using 2D Zernike moments (region shape encoding) and Fourier descriptors (contour shape encoding). The binary images are captured using a set of virtual cameras positioned on the surface of a bounding sphere. Their positioning is based on an almost-even distribution approach [23]. As LIFD encodes the 3D object's structure using images captured from multiple viewpoints is considered as a good candidate to evaluate its performance in relation to ZMDM and CBDM. In our experiments we have used the source code being provided by the authors for both the descriptor extraction and the shape similarity calculations.

In order to evaluate the performance of the three descriptors, we performed multiple queries-by-example on our calibrated 3D vessel database. The 3D models are a combination of royalty free digitised and manually modelled 3D vessels, copyrighted digitised vessels provided by other research groups, manually modelled vessels based on photographs and vessels created by our 3D vessel random generator [7][30]. All 3D models are described as triangular meshes. The digitised models have been acquired using the laser triangulation, shape from structured light and shape from silhouette techniques. The database covers several shape categories such as ancient Greek, Roman, Native American and modern pottery. The latter is considered as noise that is included in order to enhance the validity of the performance evaluation results. The content of the database was calibrated by archaeologists using a custom annotation software tool that 
allowed the annotation of each vessel with attributes such as the shape and the type of shape, the preservation state, etc.

For the performance evaluation, we have selected five ancient Greek shape categories due to their relatively high population within the database. These were Alabastron (55 objects), Amphora (96 objects), Hydria (26 objects), Lekythos (16 objects) and Psykter (48 objects). For the objective and quantitative evaluation of the descriptors we have used apart from the precision-recall graphs, five performance scalars proposed by the annual $3 D$ Shape Retrieval Contest [31]. Each performance scalar focuses on a different characteristic of the descriptor's performance and contributes towards a more complete evaluation approach.

The resolution of the depthmap images that have been used for evaluating the performance of the CBDM descriptor was $64 x 64$ pixels. Each cumulative character sequence is composed by 4,456 characters and the average time for computing the Needleman-Wunsch algorithm (similarity metric) for two vessels was 382 milliseconds on an $A M D$ Athlon at $2.2 \mathrm{Ghz}$ with $2 G B$ of $R A M$. Images of lower resolution $(8 x 8,16 x 16$ and $32 \times 32$ pixels) resulted lower shape matching performance while higher resolution images $(128 \times 128)$ required longer comparison times without providing any performance improvement. On the other hand, the $13^{\text {th }}$ order 2D Zernike moments (ZMDM) were extracted from $256 \times 256$ pixels depthmap images resulting a digital signature of effective 1,248 bytes (156 floating points with 64-bit precision). The average computation time of the L2-norm metric used for the ZMDM was 0.3 milliseconds. Furthermore, the effective data size of the LIFD descriptor was 4500 bytes ( 35 bytes for quantized Zernike moments plus 10 bytes for quantized Fourier coefficients for each binary) and the average computation time of the L1-norm metric was 45 milliseconds on the same system. 
For each vessel shape category we calculated the average precision-recall graphs by using each vessel as a query object. Then, we computed the overall average precisionrecall graphs for all shape categories (Figure 3). The same performance evaluation scenario has followed for all three descriptors. As shown in Figure 3 the precision-recall curve of all three descriptors share a similar curve. Higher precision values were achieved by using the CBMD and LIFD descriptors. Additionally, the graph depicts a similar performance between CBMD and LIFD within the first $15 \%$ of the ranking positions. Furthermore, the CBDM descriptor precision-recall curve indicates a better performance in several areas of the precision-recall graph when compared to LIFD. The similarity between the three precision-recall curves indicates that the depthmap images are able to capture the main shape features of a normalised in terms of scale, position and orientation vessel and thus the shape matching problem can be solved using $2 \mathrm{D}$ shape descriptors. Table I, depicts the average values of five performance scalars proposed in [30]. In all cases a higher value indicates superior performance.

Figure 3

Table I

The CBDM descriptor achieved the highest performance as being indicated by the $l^{\text {st }}$ tier, $2^{\text {nd }}$ tier and Average Precision (AP) scalars. On the other hand, the Cumulative Gain (CG) and Nearest Neighbour (NN) scalars indicate that a better performance is achieved when the LIFD descriptor is used. Nevertheless, for all three descriptors the $N N$ scalar remains close to 0.9 . This indicates that there is a $90 \%$ probability that the first ranked vessel will be of the same shape class like the query-object. The ZMDM had a better $N N$ behaviour in the case of Lekythos shape category while all three descriptors got the highest possible mark in the case of Psykter. The CBDM performance scalars indicate an almost similar performance when compared to LIFD. On the other hand, the ZMDM scalar performance is lower but still close to the other two descriptors. Again, the similar 
average performance scalar values indicate that the main morphological features of a vessel can be captured on two depthmap images and thus 2D shape descriptors can be used for the content based retrieval of 3D vessel replicas.

\section{Conclusions}

Content based retrieval can be considered an objective quantitative criterion for $3 \mathrm{D}$ models clustering. Its application in the cultural heritage domain might reduce significantly the time needed for a researching 3D digital content. In this work, we presented the idea of performing 3D shape matching of complete or nearly complete $3 \mathrm{D}$ vessels by encoding a pair of depthmap images using compact 2D shape descriptors. Those depthmap images depict the orthogonal projections of the outer surface relief of a vessel which has been normalised in terms of scale, position and orientation using an automated algorithm. We evaluated the performance of a $2 \mathrm{D}$ shape descriptor based on 2D Zernike moments and a variant of the descriptor proposed by Chaouch et al. [8] in relation to the performance of the LightField 3D descriptor [23]. The performance evaluation results indicate that the outer surface relief features captured by a pair of depthmap images can provide adequate shape information to perform content based retrieval of 3D pottery models. An experimental 3D pottery search engine that demonstrates the functionality of content based retrieval can be found at http://www.ipet.gr/3dpse. In the near future, we will attempt to evaluate the performance of descriptors that are composed by combinations of different 2D shape descriptors such as 2D Fourier descriptor, Krawtchouk moments and geodesic distance histograms.

\section{Acknowledgements}

This paper is part of the 03ED679 research project, implemented within the framework of the "Reinforcement Programme of Human Research Manpower" (PENED) and co financed by National and Community Funds (25\% from the Greek Ministry of Development - General 
Secretariat of Research and Technology and 75\% from E.U.- European Social Fund). The authors would like to acknowledge and thank the Cultural Heritage Unit of the Cultural and Educational Technology Institute/R.C. 'Athena' for their support in this work, Dr. Horn-yeu Shiaw, Prof. Robert Jacob and Prof. Gregory R. Crane from Tufts University, the Centre of Advanced Spatial Technologies of the Hampson Museum, Carlos Hernández Esteban and Francis Schmitt from Télécom Paris for providing us with copies of their digitised 3D vessel collections.

\section{References}

[1] M. S. Lew, N. Sebe, C. DJeraba, R. Jain, Content-Based Multimedia Information Retrieval: State of the Art and Challenges, ACM Trans. Multimedia Comput. Commun. Appl., Vol. 2(1), 2006, pp.1-19.

[2] J. W. H. Tangelder, R. C. Veltkamp, A survey of content based 3D shape retrieval methods, Multimedia Tools and Applications, Vol. 39, pp.441-471.

[3] C. Carpineto, S. Osinski, G. Romano, D. Weiss, A Surver of Web Clustering Engines, ACM Computing Surveys, Vol. 41(3), Article 17, 2009, pp. 1-38.

[4] W. R. Agualimpia, F. J. L. Pellicer, Pedro R. M. Medrano, J. N. Iso, F. J. Z. Soria, Exploring the Advances in Semantic Search Engines, Advances in Soft Computing, Vol. 79, 2010, pp.613-620.

[5] D. Gorisse, M. Cord, M. Jordan, S. Philipp-Foliguet, F. Precioso, 3D Content-Based Retrieval In Artwork Databases, Proceedings of the 3DTV-Conference, Kos Island, Greece, May 7-9, 2007, pp. 1-4.

[6] C. Hörr, G. Brunnett, Similarity Estimation on Ancient Vessels, Proceedings of the GraphiCon Conference, Moscow State University, Russia, June 23-27, 2008, pp. 94-100.

[7] A. Koutsoudis, G. Pavlidis, V. Liami, D. Tsiafaki, C. Chamzas, 3D Pottery Content Based Retrieval Based on Pose Normalisation and Segmentation, Journal of Cultural Heritage, Vol. 11(3),July-September 2010, pp. 329-338. 
[8] M. Chaouch, A. Verroust-Blondet, A New Descriptor for 2D Depth Image Indexing and 3D Model Retrieval, in Procs. of ICIP 2007, September 16-19, San Antonio, Texas, USA, pp. 373-376.

[9] D.-Y. Chen, X.-P. Tian, Y.-T. Shen, and M. Ouhyoung, On visual similarity based 3D model retrieval, Computer Graphics Forum, Vol. 22(3), September 2003, pp. 223-232.

[10] C. Maiza, V. Gaildrat, Automatic Classification of Archaeological Potsherds, Proceedings of the 8th International Conference on Computer Graphics and Artificial Intelligence, Limoges, France, May 11-12, 2005, pp. 135-147.

[11] C. Maiza, V. Gaildrat, SemanticArchaeo: A Symbolic Approach of Pottery Classification, Proceedings of the $7^{\text {th }}$ International Symposium on Virtual Reality, Nicosia, Cyprus, October 30 - November 4, 2006, pp. 227-233.

[12] Q. Huang, S. Flory, N. Gelfand, M. Hofer, H. Pottman, Reassembling Fractured Objects by Geometric Matching, ACM Trans. Graphics, Vol. 25, Issue 3, (2006) 569578.

[13] M. Kampel, H. Mara, R. Sablatnig, Robust 3D Reconstruction of Archaeological Pottery based on Concentric Circular Rills, Proceedings of the 6th International Workshop on Image Analysis for Multimedia Interactive Services, Montreux, Switzerland, April 13-15, 2005, pp. 14-20.

[14] N. Mellado, P. Reuter, C. Schlick, Semi-automatic Geometry-Drive Reassembly of Fractured Archeological Objects, Proceedings of the 11th International Symposium on Virtual Reality, Archaeology and Cultural Heritage, Paris, France, September 21-24, 2010, pp. 33-38.

[15] M. Kampel, R. Sablatnig, On 3D Mosaicing of Rotationally Symmetric Ceramic Fragments, Proceedings of the 17th International Conference on Pattern Recognition (ICPR'04), Cambridge, England, UK, August 23-26, 2004, Vol. 2, pp. 265-268. 
[16] Y. Cao, D. Mumford, Geometric Structure Estimation of Axially Symmetric Pots from Small Fragments, Proceedings of the Signal Processing, Pattern Recognition and Applications, IASTED, Crete, Greece, June 25-28, 2002, pp. 92-97.

[17] L. Dezhi, R. Anshuman, S. Arleyn, B. Myungsoo, An XML-based information model for archaeological pottery, Journal of Zhejiang University Science, Vol. 6A(5), (2005) 447-453.

[18] L. Dezhi, A. Razdan, Knowledge-Based Search Engine for Specific 3D models, Proceedings of the International Symposium on Computational and Information Science, Shanghai, China, December 16-18, 2006, pp. 530-537.

[19] A. Gilboa, A. Karasik, I. Sharon, U. Smilansky, Towards computerized typology and classification of ceramics, Journal of Archaeological Science, 31 (2004) 681-694.

[20] F. J. Melero, A. Leon, F. Contreras, J.C. Torres, A new system for interactive vessel reconstruction and drawing, Proceedings of Computer Applications in Archaeology, Vienna, Austria, April 8 - 12, 2003, pp. 8-12.

[21] S. Uppalapati, J. C. Femiani, A. Razdan, K. Gary, 3D VQI: 3D Visual Query Interface, Proceedings of the $6^{\text {th }}$ International Confernece on Information Technology: New Generations (ITNG '09), April 27-29, 2009, Las Vegas, Nevada, USA, pp. 13471354.

[22] M. Hofer, B. Odehnal, H. Pottmann, T. Steiner, J. Wallner, 3D Shape Recognition and Reconstruction Based on Line Element Geometry, Proceedings of the Tenth IEEE International Conference on Computer Vision, (ICCV'05), October 17-20, Beijing, China, 2005, pp. 1532--1538.

[23] C. M. Chyr, B. B. Kimia, 3D Object Recognition Using Shape Similarity-based Aspect Graph, Proceedings of the Eighth IEEE International Conference on Computer Vision (ICCV 2001), Vancouver, July 7-14, 2001, British Columbia, Canada, pp.254261.

[24] T. B. Sebastian, P. N. Klein, B. B. Kimia, Recognition of Shapes by Editing Shock Graphs, Proceedings of the Eighth IEEE International Conference on Computer 
Vision (ICCV 2001), Vancouver, July 7-14, 2001, British Columbia, Canada, pp.755762.

[25] N. Iyer, S. Jayanti, K. Lou, Y. Kalyanaraman, K. Ramani, Three-dimensional shape searching: state-of-the-art review and future trends, Journal of Computer-Aided Design, Vol. 37(2005), pp. 509-530.

[26] A. Khotanzand, Y. H. Hongs, Invariant image recognition by Zernike moments, IEEE Trans. on Pattern Analysis and Machine Intelligence, Vol. 12(5),1990, pp. 489-497.

D. Zhang, G. Lu, Shape-based image retrieval using generic Fourier descriptor, Signal Processing: Image Communication, Vol. 17(10), 2002, pp. 825-848.

[28] P. Daras, A. Axenopoulos, A compact multi-view descriptor for 3D object retrieval, in Procs. of the $7^{\text {th }}$ International Workshop on Content-Based Multimedia Indexing, June 3-5, 2009, Chania, Crete, Greece, pp.115-119.

[29] S. Needleman, C. Wunsch, A General Method Applicable to the Search for Similiraties in the Amino Acid Sequence of two proteins, Journal of Molecular Biology, vol. 48 , no. 3,1970 , pp. $443-453$.

[30] A. Koutsoudis, G. Pavlidis, F. Arnaoutoglou, D. Tsiafakis, C. Chamzas, qp: A Tool for Generating 3D Models of Ancient Greek Pottery, Journal of Cultural Heritage Vol. 10 (2009), pp. 281-295.

[31] 3D Shape Retrieval Contest, http://www.aimatshape.net/event/SHREC 


\section{Figures - Titles}

Figure 1 - Orthogonal projection plane topology

Figure 2 - Ancient Greek Lekythos depthmap encoding using character sequences Figure 3 - Overall Averaged Precision - Recall graph

\section{Tables}

Table I - Average values of performance scalars 


\section{Figures}
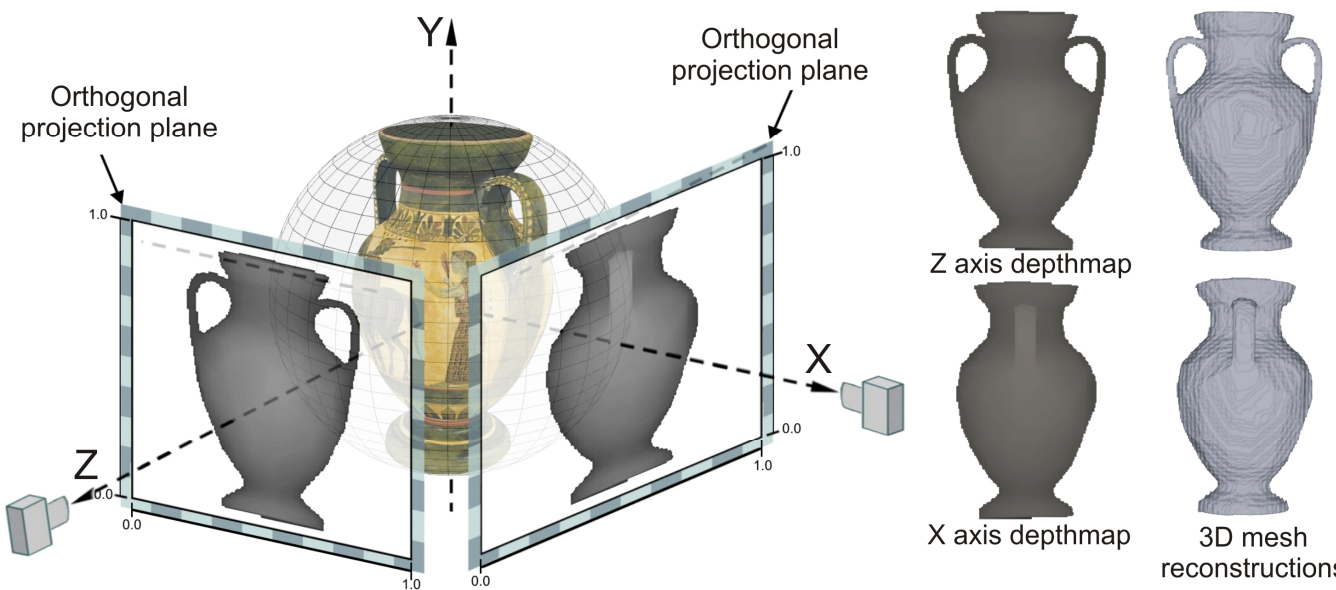

$Z$ axis depthmap
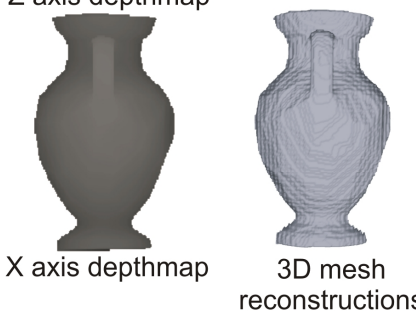

Figure 1 - Orthogonal projection plane topology

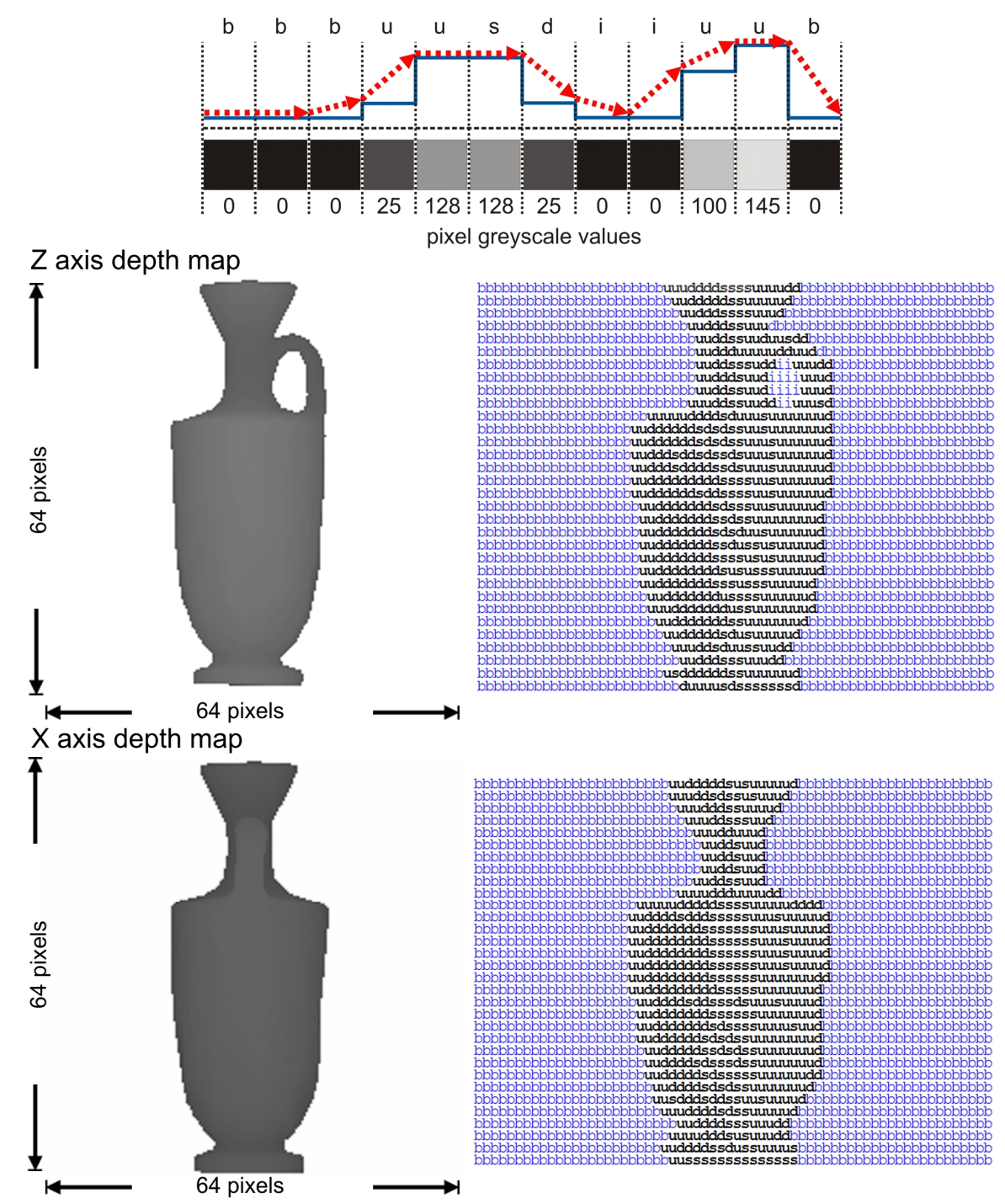

$Z$ axis depth map
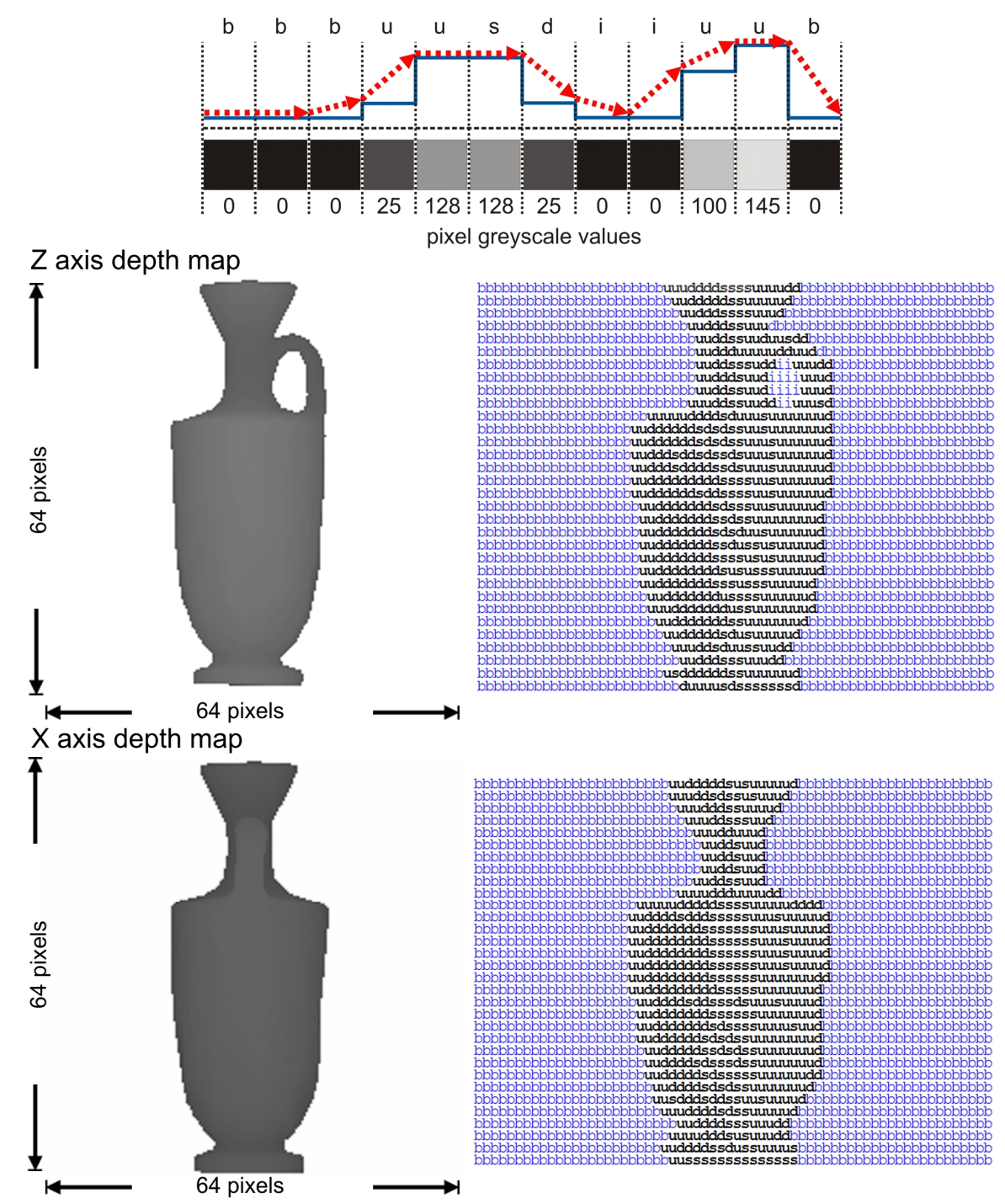

64 pixels

Figure 2 - Ancient Greek Lekythos depthmap encoding using character sequences 


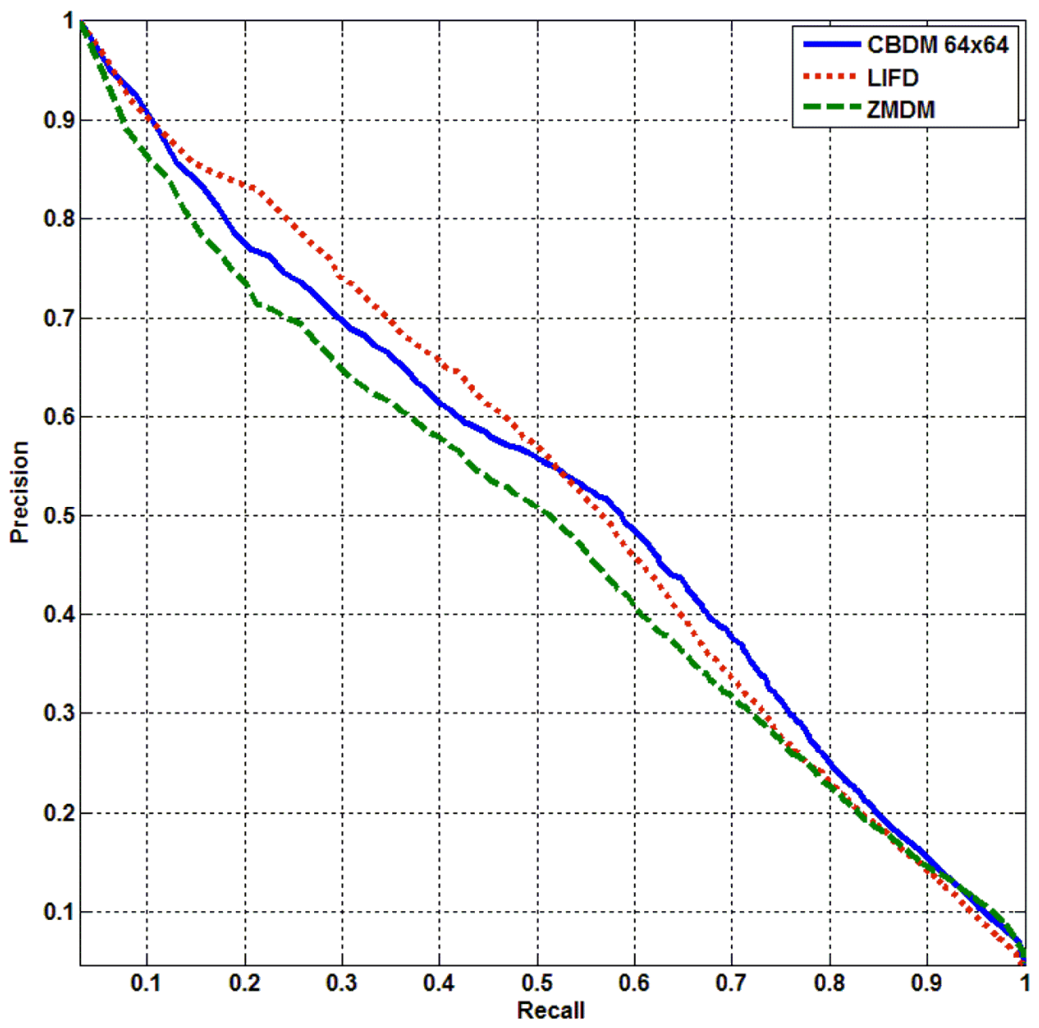

Figure 3 - Overall Average Precision-Recall graph 
Tables

\begin{tabular}{|c|c|c|c|c|c|c|c|c|c|c|c|c|c|c|c|}
\hline & \multicolumn{3}{|c|}{$1^{\text {st }} \mathrm{Tier}$} & \multicolumn{3}{|c|}{$2^{\text {nd }}$ Tier } & \multicolumn{3}{|c|}{$A P$} & \multicolumn{3}{|c|}{$C G(64)$} & \multicolumn{3}{|c|}{$N N$} \\
\hline & 总 & 全 & $\sum_{N}^{\sum}$ & 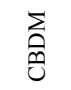 & 全 & $\sum_{N}^{\sum}$ & 总 & 仝 & $\sum_{N}^{\sum}$ & 苗 & 国 & $\sum_{N}^{\sum}$ & 音 & 妟 & $\sum_{N}^{\sum}$ \\
\hline Alabastron & 52.72 & 53.78 & 52.92 & 36.31 & 36.47 & 37.12 & 0.49 & 0.53 & 0.50 & 31.90 & 32.07 & 31.36 & 0.89 & 0.98 & 0.90 \\
\hline Amphora & 46.21 & 49.58 & 46.87 & 30.31 & 31.11 & 31.03 & 0.47 & 0.49 & 0.48 & 37.56 & 41.42 & 38.01 & 0.87 & 0.86 & 0.84 \\
\hline Hydria & 38.98 & 40.38 & 27.66 & 24.53 & 25.59 & 18.12 & 0.31 & 0.34 & 0.21 & 12.28 & 14.76 & 10.57 & 0.88 & 0.96 & 0.80 \\
\hline Lekythos & 52.34 & 51.95 & 48.82 & 31.83 & 32.42 & 32.03 & 0.41 & 0.41 & 0.40 & 13.31 & 12.37 & 13.18 & 0.87 & 0.81 & 0.87 \\
\hline Psykter & 83.98 & 72.13 & 73.78 & 44.48 & 40.95 & 42.77 & 0.79 & 0.70 & 0.72 & 41.60 & 36.95 & 38.22 & 1 & 1 & 1 \\
\hline Average & 54.85 & 53.56 & 50.01 & 33.49 & 33.31 & 32.21 & 0.50 & 0.49 & 0.46 & 27.33 & 27.52 & 26.27 & 0.90 & 0.92 & 0.88 \\
\hline
\end{tabular}

Table I - Average values of performance scalars 